status slowly improves such that she is on face-mask $\mathrm{O}_{2}$ by day 3 of her admission. She is haemodynamically stable and her urine output is satisfactory with maintenance iv fluid replacement of over $2 \mathrm{l} /$ day. Unfortunately, her serum creatinine, although initially normal, rose to $153 \mu \mathrm{mol} / \mathrm{l}$ on day 4 and continued to rise at around 50-70 $\mu \mathrm{mol} / \mathrm{l} / \mathrm{day}$.

Which of the following statements are true and which false?

(a) Her antibiotic dosing should be reviewed in line with her level of renal function

(b) Renal failure is likely to be due to septic ATN

(c) Urinalysis is indicated

(d) Renal biopsy is indicated

(e) Persistence of presumed ATN beyond 2-3 weeks should always prompt investigation for an alternative diagnosis

A 75-year-old man with type 2 diabetes mellitus, hypertension and stable angina developed a florid salmonella gastroenteritis whilst on holiday. He had failed to keep up with his fluids and had continued to take his metformin and perindopril. On admission he is clinically dehydrated, hypotensive (BP 103/64 $\mathrm{mmHg}$ ) and oliguric. His serum creatinine is $296 \mu \mathrm{mol} / \mathrm{l}$ and serum potassium $5.7 \mathrm{mmol} / \mathrm{l}$. Unfortunately, he has a witnessed cardiac arrest soon after arrival in A\&E, but was resuscitated after three cycles of basic life support and $500 \mathrm{ml}$ iv colloid resuscitation. His post-arrest BP $(96 / 53 \mathrm{mmHg})$ improved to $115 / 76 \mathrm{mmHg}$ with further fluid resuscitation but his arterial blood gases revealed a metabolic acidosis (pH 7.25, $\mathrm{BE}-8 \mathrm{mmol} / \mathrm{l}, \mathrm{PaCO}_{2} 2.6 \mathrm{kPa}$, hyperkalaemia $7.5 \mathrm{mmol} / \mathrm{l}$ and hyperlactataemia $7 \mathrm{mmol} / \mathrm{l})$. Which of the following statements are true and which false?

(a) First-line treatment should include iv calcium gluconate, iv dextrose-insulin and iv sodium bicarbonate

(b) Renal replacement therapy (RRT) is indicated upon arrival on the ICU

(c) The RRT modality of choice is continuous veno-venous haemofiltration

(d) The need for RRT establishes the diagnosis of ATN

(e) Perindopril is contraindicated lifelong, even after recovery

\section{Guidelines on completing the answer sheet for those who wish to submit their answers on paper}

A loose leaf answer sheet is enclosed, which will be marked electronically at the Royal College of Physicians. Answer sheets must be returned by 21 November 2005 to: CME Department (SAQs), Royal College of Physicians, 11 St Andrews Place, London NW1 4LE.

Overseas members only can fax their answers to 02074874156 Correct answers will be published in the next issue of Clinical Medicine.

*Further details on CME are available from the CME department at the Royal College of Physicians (address above or telephone 02079351174 extension 306 or 309).

Your completed answer sheet will be scanned to enable a quick and accurate analysis of results. To aid this process, please keep the following in mind:

1 Please print your GMC Number firmly and neatly

2 Only write in allocated areas on the form

3 Only use pens with black or dark blue ink

4 For optimum accuracy, ensure printed numbers avoid contact with box edges

5 Please shade circles like this:

6 Please mark any mistakes made like this: $\boldsymbol{X}$

7 Please do not mark any of the black squares on the corners of each page

8 Please fill in your full name and address on the back of the answer sheet in the space provided; this will be used to mail the form back to you after marking.

\title{
CME Haematology SAQs \\ Answers to the CME SAQs published in Clinical Medicine July/August 2005
}

$\begin{array}{llllllllll}\text { Q1 } & \text { Q2 } & \text { Q3 } & \text { Q4 } & \text { Q5 } & \text { Q6 } & \text { Q7 } & \text { Q8 } & \text { Q9 } & \text { Q10 } \\ \text { a) } F & \text { a) } F & \text { a) } F & \text { a) } F & \text { a) } T & \text { a) } T & \text { a) } T & \text { a) } F & \text { a) } F & \text { a) } F \\ \text { b) } F & \text { b) } T & \text { b) } F & \text { b) } T & \text { b) } F & \text { b) } F & \text { b) } T & \text { b) } T & \text { b) } T & \text { b) } F \\ \text { c) } T & \text { c) } F & \text { c) } F & \text { c) } F & \text { c) } T & \text { c) } F & \text { c) } T & \text { c) } F & \text { c) } F & \text { c) } T \\ \text { d) } T & \text { d) } F & \text { d) } F & \text { d) } T & \text { d) } F & \text { d) } T & \text { d) } F & \text { d) } T & \text { d) } T & \text { d) } F \\ \text { e) } T & \text { e) } F & \text { e) } T & \text { e) } T & \text { e) } T & \text { e) } F & \text { e) } F & \text { e) } F & \text { e) } T & \text { e) } T\end{array}$

